\title{
The Radon Inversion Problem for Holomorphic Functions in the Unit Disc
}

\author{
P. Kot ${ }^{1} \cdot$ P. Pierzchała ${ }^{1}$
}

Received: 21 March 2020 / Revised: 5 December 2020 / Accepted: 21 December 2020 /

Published online: 8 May 2021

(C) The Author(s) 2021

\begin{abstract}
This paper deals with the so-called Radon inversion problem formulated in the following way: Given a $p>0$ and a strictly positive function $H$ continuous on the unit circle $\partial \mathbb{D}$, find a function $f$ holomorphic in the unit disc $\mathbb{D}$ such that $\int_{0}^{1}|f(z t)|^{p} d t=H(z)$ for $z \in \partial \mathbb{D}$. We prove solvability of the problem under consideration. For $p=2$, a technical improvement of the main result related to convergence and divergence of certain series of Taylor coefficients is obtained.
\end{abstract}

Keywords Radon inversion problem · Holomorphic functions · Taylor series · Inner functions

Mathematics Subject Classification 30B30 · 30B10

\section{Introduction}

Generally, if $f$ is a function defined on a manifold $M$, the Radon transform assigns to $f$ its integrals over a given family of submanifolds of M. In 1917 such transformations on hyperplanes were first studied by Radon [10]. The present paper is devoted to the Radon-type transform defined on functions holomorphic in the complex unit disc that evaluates integrals of $p$-th power of the modulus of the function along radii of the unit disc. Let $\mathbb{D}$ denote the unit disc of $\mathbb{C}$ and $\mathcal{O}(\mathbb{D})$ be the space of functions holomorphic in $\mathbb{D}$. Given a $p>0$, the transform under consideration assigns to a function $f \in \mathcal{O}(\mathbb{D})$

\section{Communicated by Thomas Ransford.}

P. Pierzchała

paulina.pierzchala@agh.edu.pl; pierzchalapaulina94@gmail.com

P. Kot

piotr.kot@agh.edu.pl

1 Faculty of Applied Mathematics, AGH University of Science and Technology, al. Mickiewicza 30, 30-059 Kraków, Poland 
a function $\mathcal{R}^{p}(f): \overline{\mathbb{D}} \longmapsto \mathbb{R}_{+}$for which

$$
\mathcal{R}^{p}(f)(z):=\int_{0}^{1}|f(z t)|^{p} d t, \quad \text { where } z \in \overline{\mathbb{D}} \text {. }
$$

Then one may pose the following problem: Given a $p>0$ and a strictly positive continuous function $H$ on the unit circle $\partial \mathbb{D}$, find a holomorphic function $f \in \mathcal{O}(\mathbb{D})$ such that

$$
\mathcal{R}^{p}(f)(z)=H(z) \text { for } z \in \partial \mathbb{D} .
$$

It is worth mentioning that an almost everywhere version of the problem above was already solved by the first author in [8] even in several complex variables. However, the function $\mathcal{R}^{p}(f)$ is well-defined at all points of $\partial \mathbb{D}$. Hence, our purpose is to find a function such that (1) not only holds almost everywhere, but for all $z \in \partial \mathbb{D}$.

As was observed in [8], the problem under consideration is similar to the generalized inner function problem which in one variable is to find a function $g \in \mathcal{O}(\mathbb{D})$ such that

$$
\lim _{t \rightarrow 1}|g(z t)|=G(z) \text { for } z \in \partial \mathbb{D}
$$

given a strictly positive function $G$ continuous on $\partial \mathbb{D}$. The role of radial limits is just played by values of the function $\mathcal{R}^{p}(f)(z)$. Similarly as in [8], we intend to find a solution to (1) by constructive methods that were applied in proving the existence of a non-constant inner function in several complex variables. The main result of this paper is Theorem 6 in which we prove solvability of the Radon inversion problem. For details of the first two independent constructions of inner functions in the unit ball we refer the reader to $[1,2,9]$.

Throughout the article we shall use the following notation. Let $\sigma$ and $\mu$ stand for normalized Lebesgue measures on $\partial \mathbb{D}$ and $\overline{\mathbb{D}}$ respectively, i.e. $\sigma(\partial \mathbb{D})=1$ and $\mu(\mathbb{D})=1$. The supremum norm and $L_{p}$ norms on $\partial \mathbb{D}$ and $\mathbb{D}$ will be denoted by

$$
\begin{aligned}
& \|f\|=\sup _{z \in \overline{\mathbb{D}}}|f(z)|, \quad\|f\|_{1, \partial \mathbb{D}}=\int_{\partial \mathbb{D}}|f(z)| d \sigma(z), \\
& \|f\|_{2, \mathbb{D}}^{2}=\int_{\partial \mathbb{D}} \int_{0}^{1}|f(z t)|^{2} d t d \sigma(z) .
\end{aligned}
$$

Let $\mathcal{C}_{L}(\overline{\mathbb{D}})$ stand for the space of continuous functions in $\overline{\mathbb{D}}$ which are bounded by $L>0$.

For $p=2$, if $f=\sum_{n=0}^{\infty} a_{n} z^{n}$ satisfies (1), then we observe that

$$
\begin{aligned}
\left\|\mathcal{R}^{2}(f)\right\|_{1, \partial \mathbb{D}} & =\int_{\partial \mathbb{D}} \int_{0}^{1}\left|\sum_{n=0}^{\infty} a_{n} z^{n} t^{n}\right|^{2} d t d \sigma(z) \\
& =\sum_{n=0}^{\infty} \frac{\left|a_{n}\right|^{2}}{2 n+1}=\|H\|_{1, \partial \mathbb{D}}<\infty
\end{aligned}
$$


One may consider what happens with the series

$$
\sum_{n=0}^{\infty}\left(\frac{\left|a_{n}\right|^{2}}{2 n+1}\right)^{s}
$$

when $s \in[0,1)$. It turns out that it is possible to construct a function $f=\sum_{n=0}^{\infty} a_{n} z^{n}$ such that (1) holds and $\sum_{n=0}^{\infty}\left(\left|a_{n}\right|^{2} /(2 n+1)\right)^{s}=\infty$ for $s \in[0,1)$. This is the second main result of this paper given in Theorem 9. Our motivation to consider the above Taylor series is [4], where a construction of an analytic function in the disc algebra with a divergent series of Taylor coefficients with every power $s \in[0,2)$ is presented. For analogues in several complex variables see [5,6,12].

\section{Radon Inversion Problem for Holomorphic Functions in the Unit Disc}

For $p>0$ and $q=\min \{p, 1\}$ we may define a metric space

$$
\mathcal{H} \mathcal{R}^{p}(\mathbb{D}):=\left\{f \in \mathcal{O}(\mathbb{D}): \sup _{z \in \partial \mathbb{D}} \mathcal{R}^{p}(f)(z)<\infty\right\}
$$

with metric

$$
d(f, g):=\sup _{z \in \partial \mathbb{D}}\left(\mathcal{R}^{p}(f-g)(z)\right)^{1 / q} .
$$

Proposition 1 Space $\left(\mathcal{H} \mathcal{R}^{p}(\mathbb{D}), d\right)$ is complete for any $p>0$. Moreover, if $f_{n} \underset{n \rightarrow \infty}{\stackrel{d}{\rightarrow}} f$ in $\mathcal{H} \mathcal{R}^{p}(\mathbb{D})$, then $\mathcal{R}^{p}\left(f_{n}\right) \rightrightarrows \mathcal{R}^{p}(f)$ as $n \rightarrow \infty$ on $\partial \mathbb{D}$.

Proof Let $\left\{f_{n}\right\}_{n \in \mathbb{N}}$ be a Cauchy sequence in $\mathcal{H} \mathcal{R}^{p}(\mathbb{D})$. Set $\varepsilon>0$. There exists $N \in \mathbb{N}$ such that

$$
\left(\mathcal{R}^{p}\left(f_{m}-f_{n}\right)(z)\right)^{1 / q}<\varepsilon \text { for all } m, n \geq N, z \in \partial \mathbb{D}
$$

and hence

$$
\int_{0}^{1}\left|\left(f_{m}-f_{n}\right)(z t)\right|^{p} d t<\varepsilon^{q} \text { for all } m, n \geq N, z \in \partial \mathbb{D} \text {. }
$$

Integration over $\partial \mathbb{D}$ gives

$$
\int_{\partial \mathbb{D}} \int_{0}^{1}\left|\left(f_{m}-f_{n}\right)(z t)\right|^{p} d t d \sigma(z)<\varepsilon^{q} \text { for all } m, n \geq N .
$$

Since $f_{m}-f_{n}$ is a holomorphic function, on the basis of [11, Prop. 1.5.4.], $\mid f_{m}-$ $\left.f_{n}\right|^{p}$ is a subharmonic function. Let $K$ be a compact subset of $\mathbb{D}, w \in K$ and $r=$ 
$\operatorname{dist}(K, \partial \mathbb{D}) / 2$, so that $\overline{\mathbb{D}}(w, r) \subset \mathbb{D}$. By applying the sub-mean-value theorem to the function $\left|f_{m}-f_{n}\right|^{p}$ and $\overline{\mathbb{D}}(w, r)$, we get for all $m, n \geq N$

$$
\begin{aligned}
\left|\left(f_{m}-f_{n}\right)(w)\right|^{p} & \leq \frac{1}{\mu(\overline{\mathbb{D}}(w, r))} \int_{\mathbb{D}(w, r)}\left|\left(f_{m}-f_{n}\right)(\zeta)\right|^{p} d \mu(\zeta) \\
& \leq \frac{1}{r^{2}} \int_{\partial \mathbb{D}} \int_{0}^{1}\left|\left(f_{m}-f_{n}\right)(z t)\right|^{p} d t d \sigma(z) \\
& <\frac{\varepsilon^{q}}{r^{2}} .
\end{aligned}
$$

In particular,

$$
\sup _{w \in K}\left|\left(f_{m}-f_{n}\right)(w)\right|^{p}<\frac{\varepsilon^{q}}{r^{2}} \text { for all } m, n \geq N .
$$

This implies that $\left\{f_{n}\right\}_{n \in \mathbb{N}}$ converges locally uniformly on compact subsets of $\mathbb{D}$. Let $f$ be the limit function for $\left\{f_{n}\right\}_{n \in \mathbb{N}}$. Since $f_{n}$ are holomorphic, so is $f$.

Now we shall show that $f_{n} \stackrel{d}{\longrightarrow} f$ and $f \in \mathcal{H} \mathcal{R}^{p}(\mathbb{D})$. By (3),

$$
\int_{0}^{\delta}\left|\left(f_{m}-f_{n}\right)(z t)\right|^{p} d t<\varepsilon^{q} \quad \text { for all } \delta \in(0,1) m, n \geq N, z \in \partial \mathbb{D} .
$$

Since $f_{m}$ converges to $f$ locally uniformly, we may pass with $m$ to the limit in (4) to obtain

$$
\int_{0}^{\delta}\left|\left(f-f_{n}\right)(z t)\right|^{p} d t \leq \varepsilon^{q} \text { for all } \delta \in(0,1) n \geq N, z \in \partial \mathbb{D} .
$$

The above inequality holds for any $\delta \in(0,1)$, so

$$
\int_{0}^{1}\left|\left(f-f_{n}\right)(z t)\right|^{p} d t \leq \varepsilon^{q} \quad \text { for all } n \geq N z \in \partial \mathbb{D}
$$

Therefore, $f_{n} \underset{n \rightarrow \infty}{\stackrel{d}{\rightarrow}} f$. Moreover, by the triangle inequality, for all $n \geq N$

$$
\sup _{z \in \partial \mathbb{D}}\left(\mathcal{R}^{p}(f)(z)\right)^{1 / q} \leq \sup _{z \in \partial \mathbb{D}}\left(\mathcal{R}^{p}\left(f-f_{n}\right)(z)\right)^{1 / q}+\sup _{z \in \partial \mathbb{D}}\left(\mathcal{R}^{p}\left(f_{n}\right)(z)\right)^{1 / q}<\infty,
$$

which implies that $f \in \mathcal{H} \mathcal{R}^{p}(\mathbb{D})$.

If $f_{n} \underset{n \rightarrow \infty}{\stackrel{d}{\rightarrow}} f$, then $d\left(f, f_{n}\right) \underset{n \rightarrow \infty}{\longrightarrow} 0$. Finally, by the triangle inequality,

$$
\sup _{z \in \partial \mathbb{D}}\left(\left(\mathcal{R}^{p}(f)(z)\right)^{1 / q}-\left(\mathcal{R}^{p}\left(f_{n}\right)(z)\right)^{1 / q}\right) \leq \sup _{z \in \partial \mathbb{D}}\left(\mathcal{R}^{p}\left(f-f_{n}\right)(z)\right)^{1 / q} \underset{n \rightarrow \infty}{\longrightarrow} 0 .
$$

Thus, $\left(\mathcal{R}^{p}\left(f_{n}\right)\right)^{1 / q} \rightrightarrows\left(\mathcal{R}^{p}(f)\right)^{1 / q}$ as $n \rightarrow \infty$ and consequently $\mathcal{R}^{p}\left(f_{n}\right) \rightrightarrows \mathcal{R}^{p}(f)$ as $n \rightarrow \infty$ on $\partial \mathbb{D}$. 
Theorem 2 Fix $p>0$. Let $f \in \mathcal{C}(\mathbb{D})$. If $\mathcal{R}^{p}(f) \in \mathcal{C}(\partial \mathbb{D})$, then $\mathcal{R}^{p}(f) \in \mathcal{C}(\overline{\mathbb{D}})$.

Proof Consider a sequence of functions $\phi_{n}(z):=\int_{0}^{a_{n}}|f(z t)|^{p} d t$, where $\left\{a_{n}\right\}_{n \in \mathbb{N}}$ is a sequence increasing to 1 and $z \in \partial \mathbb{D}$. Since $f$ is continuous in $\mathbb{D},\left\{\phi_{n}(z)\right\}_{n \in \mathbb{N}}$ is an increasing sequence of continuous functions on $\partial \mathbb{D}$. Moreover,

$$
\lim _{n \rightarrow \infty} \phi_{n}(z)=\mathcal{R}^{p}(f)(z) \text { for every } z \in \overline{\mathbb{D}}
$$

If $\mathcal{R}^{p}(f)$ is continuous on $\partial \mathbb{D}$, then by Dini's theorem $\phi_{n}$ converges uniformly to $\mathcal{R}^{p}(f)$ on $\partial \mathbb{D}$.

Fix $\varepsilon>0$. By continuity of $\mathcal{R}^{p}(f)$ on $\partial \mathbb{D}$, there exists $\delta>0$ such that for any $z, \bar{z} \in \partial \mathbb{D}$ if $|z-\bar{z}| \leq 2 \delta$, then

$$
\begin{aligned}
& \text { - }\left|\mathcal{R}^{p}(f)(z)-\mathcal{R}^{p}(f)(\bar{z})\right| \leq \frac{\varepsilon}{3} \\
& \text { - } \frac{1}{1-\delta} \int_{1-\delta}^{1}|f(z t)|^{p} d t \leq \frac{\varepsilon}{3} \text { for } z \in \partial \mathbb{D} \\
& \text { - }\left(\frac{1}{1-\delta}-1\right) \int_{0}^{1}|f(z t)|^{p} d t \leq \frac{\varepsilon}{3} \text { for } z \in \partial \mathbb{D} .
\end{aligned}
$$

Let $|z-w| \leq \delta$, where $w \in \mathbb{D}, z \in \partial \mathbb{D}$. Then there exists $w^{*} \in \partial \mathbb{D}$ such that $w=s w^{*}$ for some $s \in(1-\delta, 1)$. Hence we may estimate

$$
\begin{aligned}
\left|\mathcal{R}^{p}(f)\left(w^{*}\right)-\mathcal{R}^{p}(f)(w)\right| & \\
& =\left.\left|\int_{0}^{1}\right| f\left(w^{*} t\right)\right|^{p} d t-\int_{0}^{1}|f(w t)|^{p} d t \mid \\
& =\left.\left|\int_{0}^{1}\right| f\left(w^{*} t\right)\right|^{p} d t-\int_{0}^{1}\left|f\left(w^{*} s t\right)\right|^{p} d t \mid \\
& =\left.\left|\int_{0}^{1}\right| f\left(w^{*} t\right)\right|^{p} d t-\frac{1}{s} \int_{0}^{s}\left|f\left(w^{*} t\right)\right|^{p} d t \mid \\
& \leq\left.\left|\int_{0}^{1}\right| f\left(w^{*} t\right)\right|^{p} d t-\left.\frac{1}{s} \int_{0}^{1}\left|f\left(w^{*} t\right)\right|^{p} d t\left|+\frac{1}{s} \int_{s}^{1}\right| f\left(w^{*} t\right)\right|^{p} d t \\
& \leq\left(\frac{1}{1-\delta}-1\right) \int_{0}^{1}\left|f\left(w^{*} t\right)\right|^{p} d t+\frac{1}{1-\delta} \int_{1-\delta}^{1}\left|f\left(w^{*} t\right)\right|^{p} d t \\
& \leq \frac{2 \varepsilon}{3} .
\end{aligned}
$$

Finally, observe that by the triangle inequality, $\left|z-w^{*}\right| \leq|z-w|+\left|w-w^{*}\right| \leq 2 \delta$. Therefore,

$$
\begin{aligned}
\left|\mathcal{R}^{p}(f)(z)-\mathcal{R}^{p}(f)(w)\right| & \leq\left|\mathcal{R}^{p}(f)(z)-\mathcal{R}^{p}(f)\left(w^{*}\right)\right|+\left|\mathcal{R}^{p}(f)\left(w^{*}\right)-\mathcal{R}^{p}(f)(w)\right| \\
& \leq \frac{\varepsilon}{3}+\frac{2 \varepsilon}{3}=\varepsilon
\end{aligned}
$$

for $|z-w| \leq \delta$.

This theorem implies immediately that for any solution $g$ to the Radon inversion problem, the function $\mathcal{R}^{p}(g)$ is continuous in $\overline{\mathbb{D}}$. 
Lemma 3 Fix $p>0$. Let $H$ be a strictly positive continuous function on $\partial \mathbb{D}$. For every $\varepsilon_{1}, \varepsilon_{2} \in(0,1)$ and compact set $K \subset \mathbb{D}$ there exist $N \in \mathbb{N}$ and a polynomial $Q$ such that $Q \neq 0$ on $\partial \mathbb{D}$ and if $f_{n}(z)=(n p+1)^{1 / p} Q(z) z^{n}$, then for $n \geq N$ the following conditions are satisfied

1. $\left|f_{n}(z)\right| \leq \varepsilon_{1}$ for $z \in K$

2. $H(z)\left(1-\varepsilon_{2}\right)<\mathcal{R}^{p}\left(f_{n}\right)(z)<H(z)\left(1+\varepsilon_{2}\right)$ for $z \in \partial \mathbb{D}$.

Proof Due to the fact that every continuous function can be approximated by a function of class $\mathcal{C}^{\infty}$, there exists a strictly positive function $v \in \mathcal{C}^{\infty}(\partial \mathbb{D})$ such that

$$
v(z)\left(1-\frac{\varepsilon_{2}}{5}\right) \leq H(z) \leq v(z)\left(1+\frac{\varepsilon_{2}}{5}\right) \text { for } z \in \partial \mathbb{D} .
$$

Also, there exists a function $u$ such that $v(z)=e^{p u(z)}$ for $z \in \partial \mathbb{D}$. Since $v \in$ $\mathcal{C}^{\infty}(\partial \mathbb{D}), u$ satisfies Hölder condition. Let $\mathcal{S}(u)$ denote the Schwarz integral of $u$. By its properties, $\operatorname{Re}(\mathcal{S}(u))=u$ on $\partial \mathbb{D}$ and $\mathcal{S}(u)$ is continuous in $\overline{\mathbb{D}}$ (see [3,7]). Hence, there exists $r \in(0,1)$ such that

$$
e^{p u(z)}\left(1-\frac{\varepsilon_{2}}{5}\right) \leq e^{p \operatorname{Re}(\mathcal{S}(u)(t z))} \leq e^{p u(z)}\left(1+\frac{\varepsilon_{2}}{5}\right) \text { for } t \in[r, 1] \text { and } z \in \partial \mathbb{D} .
$$

There exists a polynomial $Q(z)$ that approximates the function $e^{\mathcal{S}(u)(z)}$ in the following way

$$
\left(1-\frac{\varepsilon_{2}}{5}\right) e^{p \operatorname{Re}(\mathcal{S}(u)(z))} \leq|Q(z)|^{p} \leq\left(1+\frac{\varepsilon_{2}}{5}\right) e^{p \operatorname{Re}(\mathcal{S}(u)(z))} \quad \text { for } z \in \overline{\mathbb{D}} .
$$

Let $M:=\sup _{z \in \partial \mathbb{D}} H(z)$. The maximum principle implies that

$$
\sup _{z \in \overline{\mathbb{D}}} e^{p \operatorname{Re}(\mathcal{S}(u)(z))}=\sup _{z \in \partial \mathbb{D}} e^{p u(z)}=\sup _{z \in \partial \mathbb{D}} v(z) \stackrel{(5)}{\leq} \frac{5}{5-\varepsilon_{2}} \sup _{z \in \partial \mathbb{D}} H(z)=\frac{5 M}{5-\varepsilon_{2}} .
$$

There exists a constant $C>0$ such that $5 M /\left(5-\varepsilon_{2}\right) \leq C H(z)$ for $z \in \partial \mathbb{D}$. Choose $N_{1} \in \mathbb{N}$ such that $r^{n p+1} \leq \varepsilon_{2} /(5 C)$ for all $n \geq N_{1}$. Then consider a polynomial $f_{n}(z)=(n p+1)^{1 / p} Q(z) z^{n}$, where $n \geq N_{1}$. For $z \in \partial \mathbb{D}$ and $n \geq N_{1}$ the above inequalities give the required estimation

$$
\begin{aligned}
\int_{0}^{1}\left|f_{n}(z t)\right|^{p} d t & \geq(n p+1) \int_{r}^{1}|Q(z t)|^{p} t^{n p} d t \stackrel{(7)}{\geq}\left(1-\frac{\varepsilon_{2}}{5}\right)(n p+1) \int_{r}^{1} e^{p \operatorname{Re}(\mathcal{S}(u)(z t))} t^{n p} d t \\
& \stackrel{(6)}{\geq} e^{p u(z)}\left(1-\frac{\varepsilon_{2}}{5}\right)^{2}(n p+1) \int_{r}^{1} t^{n p} d t \stackrel{(5)}{\geq} H(z)\left(1-\frac{\varepsilon_{2}}{5}\right)^{3}\left(1-r^{n p+1}\right) \\
& \geq H(z)\left(1-\frac{\varepsilon_{2}}{5}\right)^{4} \geq H(z)\left(1-\varepsilon_{2}\right)
\end{aligned}
$$

and

$$
\int_{0}^{1}\left|f_{n}(z t)\right|^{p} d t=(n p+1) \int_{0}^{1}|Q(z t)|^{p} t^{n p} d t
$$




$$
\begin{aligned}
& \stackrel{\text { (7) }}{\leq}\left(1+\frac{\varepsilon_{2}}{5}\right)(n p+1) \int_{0}^{1} e^{p \operatorname{Re}(\mathcal{S}(u)(z t))} t^{n p} d t \\
& \stackrel{(6)}{\leq}(n p+1)\left(1+\frac{\varepsilon_{2}}{5}\right)\left(\frac{5 M}{5-\varepsilon_{2}} \int_{0}^{r} t^{n p} d t+\left(1+\frac{\varepsilon_{2}}{5}\right) e^{p u(z)} \int_{r}^{1} t^{n p} d t\right) \\
& \stackrel{(5)}{\leq} \frac{5 M}{5-\varepsilon_{2}}\left(1+\frac{\varepsilon_{2}}{5}\right) r^{n p+1}+H(z)\left(1+\frac{\varepsilon_{2}}{5}\right)^{3}\left(1-r^{n p+1}\right) \\
& \stackrel{(8)}{\leq} H(z)\left(1+\frac{\varepsilon_{2}}{5}\right) C r^{n p+1}+H(z)\left(1+\frac{\varepsilon_{2}}{5}\right)^{3}\left(1-r^{n p+1}\right) \\
& \leq H(z)\left(1+\frac{\varepsilon_{2}}{5}\right)\left(C r^{n p+1}+\left(1+\frac{\varepsilon_{2}}{5}\right)^{2}\right) \\
& \leq H(z)\left(1+\varepsilon_{2}\right) .
\end{aligned}
$$

Now, to make $f_{n}$ satisfy the statement 1 it is enough to select a natural number $N_{2} \geq N_{1}$ such that for all $n \geq N_{2}$

$$
\left|f_{n}(z)\right|=(n p+1)^{1 / p}|Q(z)||z|^{n} \leq \varepsilon_{1} \text { for } z \in K \text {. }
$$

Finally, observe that the function $f_{n}$ constructed above, where $n$ is sufficiently large, has the required properties.

Remark 4 We can replace the statement 2 in Lemma 3 by one of the following $2^{\prime} . H(z)\left(1-\varepsilon_{2}\right)<\mathcal{R}^{p}(f)(z)<H(z)$ for $z \in \partial \mathbb{D}$ $2^{\prime \prime} . H(z)-\varepsilon_{2}<\mathcal{R}^{p}(f)(z)<H(z)$ for $z \in \partial \mathbb{D}$.

Proof For statement $2^{\prime}$ it is enough to take $\tilde{H}(z):=H(z) /\left(1-\tilde{\varepsilon}_{2}\right)$ and $\tilde{\varepsilon}_{2}:=\varepsilon_{2} /(2-$ $\left.\varepsilon_{2}\right)$ in Lemma 3. To obtain conclusion $2^{\prime \prime}$ we apply the lemma above with $\hat{\varepsilon}_{2}:=\tilde{\varepsilon}_{2} / M$, where $M:=\sup _{z \in \partial \mathbb{D}}|H(z)|$.

Lemma 5 Fix $p>0$. Let $p>0$ and $Q$ be a polynomial such that $Q(z) \neq 0$ for $z \in \partial \mathbb{D}$. For every $\varepsilon_{3} \in(0,1)$ there exists $N \in \mathbb{N}$ such that for $n \geq N$ and all functions $g \in \mathcal{C}_{L}(\overline{\mathbb{D}})$ the function $f_{n}(z)=(n p+1)^{1 / p} Q(z) z^{n}$ satisfies

$$
\mathcal{R}^{p}\left(f_{n}\right)(z)+\mathcal{R}^{p}(g)(z)-\varepsilon_{3}<\mathcal{R}^{p}\left(f_{n}+g\right)(z)<\mathcal{R}^{p}\left(f_{n}\right)(z)+\mathcal{R}^{p}(g)(z)+\varepsilon_{3} .
$$

Proof Choose $k \in \mathbb{N}$ such that $k \geq p$. First, by triangle inequality, observe that

$$
\begin{aligned}
\mathcal{R}^{p / k}\left(f_{n}^{k}+g^{k}\right)(z)= & \int_{0}^{1}\left|\left(f_{n}^{k}+g^{k}\right)(z t)\right|^{p / k} d t \leq \int_{0}^{1}\left|f_{n}(z t)\right|^{p} d t \\
& +\int_{0}^{1}|g(z t)|^{p} d t=\mathcal{R}^{p}\left(f_{n}\right)(z)+\mathcal{R}^{p}(g)(z) .
\end{aligned}
$$

Let $\delta \in(0,1)$ be such that $\int_{\delta}^{1}|g(z t)|^{p}<\varepsilon_{3} / 8$. Since

$$
\int_{0}^{\delta}\left|f_{n}(z t)\right|^{p} d t \leq(n p+1)\|Q\|^{p} \int_{0}^{\delta} t^{n p} d t=\|Q\|^{p} \delta^{n p+1},
$$


there exists $N_{1} \in \mathbb{N}$ such that $\int_{0}^{\delta}\left|f_{n}(z t)\right|^{p} d t<\varepsilon_{3} / 8$ for all $n \geq N_{1}$. Thus, the above inequalities imply the following

$$
\begin{aligned}
\mathcal{R}^{p / k}\left(f_{n}^{k}+g^{k}\right)(z) & =\int_{0}^{1}\left|\left(f_{n}^{k}+g^{k}\right)(z t)\right|^{p / k} d t \\
& =\int_{0}^{\delta}\left|\left(f_{n}^{k}+g^{k}\right)(z t)\right|^{p / k} d t+\int_{\delta}^{1}\left|\left(f_{n}^{k}+g^{k}\right)(z t)\right|^{p / k} d t \\
& \geq \int_{0}^{\delta}|g(z t)|^{p} d t-\int_{0}^{\delta}\left|f_{n}(z t)\right|^{p} d t+\int_{\delta}^{1}\left|f_{n}(z t)\right|^{p} d t-\int_{\delta}^{1}|g(z t)|^{p} d t \\
& =\mathcal{R}^{p}\left(f_{n}\right)(z)+\mathcal{R}^{p}(g)(z)-2 \int_{0}^{\delta}\left|f_{n}(z t)\right|^{p} d t-2 \int_{\delta}^{1}|g(z t)|^{p} d t \\
& >\mathcal{R}^{p}\left(f_{n}\right)(z)+\mathcal{R}^{p}(g)(z)-\frac{\varepsilon 3}{2} .
\end{aligned}
$$

If $0<p \leq 1$, then $k=1$ does the job. For $p>1$ we need a little bit more effort to accomplish this. Denote

$$
\mathcal{I}_{k}^{p}\left(f_{n}, g\right)(z):=\int_{0}^{1}\left|\sum_{m=1}^{k-1}\left(\begin{array}{c}
k \\
m
\end{array}\right)\left(f_{n}(z t)\right)^{m}(g(z t))^{k-m}\right|^{p / k} d t
$$

and notice that

$$
\begin{aligned}
\mathcal{I}_{k}^{p}\left(f_{n}, g\right)(z) & \leq \sum_{m=1}^{k-1}\left(\begin{array}{c}
k \\
m
\end{array}\right)^{p / k} \int_{0}^{1}\left|f_{n}(z t)\right|^{m p / k}|g(z t)|^{(k-m) p / k} d t \\
& \leq \sum_{m=1}^{k-1}\left(\begin{array}{c}
k \\
m
\end{array}\right)^{p / k}\|Q\|^{m p / k} L^{p(k-m) / k}(n p+1)^{m / k} \int_{0}^{1} t^{n m p / k} d t \\
& =\sum_{\substack{k-1 \\
\rightarrow=1}}^{\rightarrow}\left(\begin{array}{c}
k \\
m
\end{array}\right)^{p / k}\|Q\|^{m p / k} L^{p(k-m) / k} \frac{k(n p+1)^{m / k}}{n m p+k}
\end{aligned}
$$

Therefore, there exists $N_{2} \in \mathbb{N}$ such that $\mathcal{I}_{k}^{p}\left(f_{n}, g\right)(z)<\varepsilon_{3} / 2$ for $n \geq N_{2}$. This yields the conclusion rather quickly 


$$
\begin{aligned}
\mathcal{R}^{p}\left(f_{n}+g\right)(z) & =\int_{0}^{1}\left|\left(f_{n}+g\right)(z t)\right|^{p} d t \\
& =\int_{0}^{1}\left(\left|\left(f_{n}+g\right)(z t)\right|^{k}\right)^{p / k} d t \\
& =\int_{0}^{1}\left|\sum_{m=0}^{k}\left(\begin{array}{c}
k \\
m
\end{array}\right)\left(f_{n}(z t)\right)^{m}(g(z t))^{k-m}\right|^{p / k} d t \\
& \leq \int_{0}^{1}\left|f_{n}(z t)\right|^{p} d t+\int_{0}^{1}|g(z t)|^{p} d t+\mathcal{I}_{k}^{p}\left(f_{n}, g\right)(z) \\
& <\mathcal{R}^{p}\left(f_{n}\right)(z)+\mathcal{R}^{p}(g)(z)+\frac{\varepsilon_{3}}{2}
\end{aligned}
$$

and

$$
\begin{aligned}
\mathcal{R}^{p}\left(f_{n}+g\right)(z) & =\int_{0}^{1}\left|\left(f_{n}+g\right)(z t)\right|^{p} d t \\
& =\int_{0}^{1}\left|\sum_{m=0}^{k}\left(\begin{array}{c}
k \\
m
\end{array}\right)\left(f_{n}(z t)\right)^{m}(g(z t))^{k-m}\right|^{p / k} d t \\
& \geq \int_{0}^{1}\left|\left(f_{n}^{k}+g^{k}\right)(z t)\right|^{p / k} d t-\mathcal{I}_{k}^{p}\left(f_{n}, g\right)(z) \\
& >\int_{0}^{1}\left|f_{n}(z t)\right|^{p} d t+\int_{0}^{1}|g(z t)|^{p} d t-\frac{\varepsilon_{3}}{2}-\frac{\varepsilon_{3}}{2} \\
& =\mathcal{R}^{p}\left(f_{n}\right)(z)+\mathcal{R}^{p}(g)(z)-\varepsilon_{3} .
\end{aligned}
$$

Theorem 6 Fix $p>0$. Let $G$ be a strictly positive continuous function defined on $\partial \mathbb{D}$. There exists a function $f \in \mathcal{O}(\mathbb{D})$ such that

$$
\mathcal{R}^{p}(f)(z)=G(z) \text { for } z \in \partial \mathbb{D} .
$$

Moreover, $\mathcal{R}^{p}(f) \in \mathcal{C}(\overline{\mathbb{D}})$.

Proof Since $G$ is strictly positive, there exists $m \in \mathbb{N}$ such that $G(z)-2^{-m}>0$ for all $z \in \partial \mathbb{D}$. We will apply Lemmas 3 and 5 iteratively to construct a sequence $\left\{f_{n}\right\}_{n \in \mathbb{N}}$ of functions in the disc algebra and a sequence of real numbers $0<r_{0} \leq r_{1} \leq \cdots \leq$ $r_{n} \leq r_{n+1} \leq \cdots \leq 1$ satisfying the following conditions

(a) $\left|f_{n}(z)\right| \leq 2^{-n}$ for $z \in \overline{\mathbb{D}}_{r_{n-1}}$

(b) $\mathcal{R}^{p}\left(\sum_{k=1}^{n} f_{k}\right)(z)<G(z)-2^{-(m+n+2)}$ for $z \in \partial \mathbb{D}$

(c) $\int_{0}^{r_{n}}\left|\sum_{k=1}^{n} f_{k}(z t)\right|^{p} d t>G(z)-2^{-(m+n)}$ for $z \in \partial \mathbb{D}$.

We begin by selecting $r_{0}=1 / 2$ and applying Lemma 3 in version $2^{\prime \prime}$ with

$$
H:=G-2^{-(m+3)}, \quad K:=\overline{\mathbb{D}}_{r_{0}}, \quad \varepsilon_{1}:=\frac{1}{2}, \quad \varepsilon_{2}:=2^{-(m+2)}
$$


to obtain a function $f_{1} \in \mathcal{O}(\mathbb{D}) \cap \mathcal{C}^{\infty}(\overline{\mathbb{D}})$ such that

- $\left|f_{1}(z)\right| \leq 1 / 2$ for $z \in \overline{\mathbb{D}}_{1 / 2}$

- $G(z)-2^{-(m+1)}<\mathcal{R}^{p}\left(f_{1}\right)(z)<G(z)-2^{-(m+3)}$ for $z \in \partial \mathbb{D}$.

Then choose $1 \geq r_{1} \geq\left(1+r_{0}\right) / 2$ such that

$$
\int_{0}^{r_{1}}\left|f_{1}(z t)\right|^{p} d t>G(z)-2^{-(m+1)} \text { for } z \in \partial \mathbb{D} .
$$

Proceeding inductively, let us assume that numbers $r_{0} \leq r_{1} \leq r_{2} \leq \cdots r_{n} \leq 1$ and functions $f_{1}, f_{2}, \ldots, f_{n} \in \mathcal{O}(\mathbb{D}) \cap \mathcal{C}^{\infty}(\overline{\mathbb{D}})$ with properties a) $-\mathrm{c}$ ) have been found. We may apply Lemmas 3 and 5 with

$$
\begin{array}{r}
H:=G-\mathcal{R}^{p}\left(\sum_{k=1}^{n} f_{k}\right)-2^{-(m+n+2)}, \quad g(z):=\sum_{k=1}^{n} f_{k}(z), \quad K:=\overline{\mathbb{D}}_{r_{n}}, \\
\varepsilon_{1}:=2^{-(n+1)}, \quad \varepsilon_{2}:=2^{-(m+n+3)}, \quad \varepsilon_{3}:=2^{-(m+n+3)}
\end{array}
$$

to produce a new function $f_{n+1} \in \mathcal{O}(\mathbb{D}) \cap \mathcal{C}^{\infty}(\overline{\mathbb{D}})$ such that the following statements hold

- $\left|f_{n+1}(z)\right| \leq 2^{-(n+1)}$ for $z \in \overline{\mathbb{D}}_{r_{n}}$

- $\mathcal{R}^{p}\left(f_{n+1}\right)>G-\mathcal{R}^{p}\left(\sum_{k=1}^{n} f_{k}\right)-2^{-(m+n+2)}-2^{-(m+n+3)}$ on $\partial \mathbb{D}$

- $\mathcal{R}^{p}\left(f_{n+1}\right)<G-\mathcal{R}^{p}\left(\sum_{k=1}^{n} f_{k}\right)-2^{-(m+n+2)}$ on $\partial \mathbb{D}$

- $\mathcal{R}^{p}\left(\sum_{k=1}^{n+1} f_{k}\right)>\mathcal{R}^{p}\left(f_{n+1}\right)+\mathcal{R}^{p}\left(\sum_{k=1}^{n} f_{k}\right)-2^{-(m+n+3)}$ on $\partial \mathbb{D}$

- $\mathcal{R}^{p}\left(\sum_{k=1}^{n+1} f_{k}\right)<\mathcal{R}^{p}\left(f_{n+1}\right)+\mathcal{R}^{p}\left(\sum_{k=1}^{n} f_{k}\right)+2^{-(m+n+3)}$ on $\partial \mathbb{D}$.

This gives on $\partial \mathbb{D}$

$$
\begin{aligned}
\mathcal{R}^{p}\left(\sum_{k=1}^{n+1} f_{k}\right) & <\mathcal{R}^{p}\left(\sum_{k=1}^{n} f_{k}\right)+\mathcal{R}^{p}\left(f_{n+1}\right)+2^{-(m+n+3)} \\
& <\mathcal{R}^{p}\left(\sum_{k=1}^{n} f_{k}\right)+G-\mathcal{R}^{p}\left(\sum_{k=1}^{n} f_{k}\right)-2^{-(m+n+2)}+2^{-(m+n+3)} \\
& =G-2^{-(m+n+3)}
\end{aligned}
$$


and

$$
\begin{aligned}
\mathcal{R}^{p}\left(\sum_{k=1}^{n+1} f_{k}\right) & >\mathcal{R}^{p}\left(\sum_{k=1}^{n} f_{k}\right)+\mathcal{R}^{p}\left(f_{n+1}\right)-2^{-(m+n+3)} \\
& >\mathcal{R}^{p}\left(\sum_{k=1}^{n} f_{k}\right)+G-\mathcal{R}^{p}\left(\sum_{k=1}^{n} f_{k}\right)-2^{-(m+n+1)} \\
& =G-2^{-(m+n+1)} .
\end{aligned}
$$

Next select $1 \geq r_{n+1} \geq\left(1+r_{n}\right) / 2$ such that

$$
\int_{0}^{r_{n+1}}\left|\sum_{k=1}^{n+1} f_{k}(z t)\right|^{p} d t>G(z)-2^{-(m+n+1)}
$$

and the induction process is complete.

By statement a), the sequence $\sum_{k=1}^{n} f_{k}$ converges uniformly to the function $f:=$ $\sum_{k=1}^{\infty} f_{k}$ on compact subsets of $\mathbb{D}$ and $f \in \mathcal{O}(\mathbb{D})$. Then notice that for all $r \in(0,1)$ and $z \in \partial \mathbb{D}$ we have

$$
\begin{aligned}
\int_{0}^{r}|f(z t)|^{p} d t & =\int_{0}^{r} \lim _{n \rightarrow \infty}\left|\sum_{k=1}^{n} f_{k}(z t)\right|^{p} d t=\lim _{n \rightarrow \infty} \int_{0}^{r}\left|\sum_{k=1}^{n} f_{k}(z t)\right|^{p} d t \\
& \leq \lim _{n \rightarrow \infty}\left(G(z)-2^{-(m+n+2)}\right) \\
& =G(z) .
\end{aligned}
$$

Hence,

$$
\mathcal{R}^{p}(f)(z) \leq G(z) \text { for } z \in \partial \mathbb{D} \text {. }
$$

Let $q=\max \{p, 1\}$. For a given $n \in \mathbb{N}$ and all $z \in \partial \mathbb{D}$ from conditions a) and c) it follows that

$$
\begin{aligned}
\left(\mathcal{R}^{p}(f)(z)\right)^{1 / q} & \geq\left(\int_{0}^{r_{n}}\left|\sum_{k=1}^{\infty} f_{k}(z t)\right|^{p} d t\right)^{1 / q} \\
& \geq\left(\int_{0}^{r_{n}}\left|\sum_{k=1}^{n} f_{k}(z t)\right|^{p} d t\right)^{1 / q}-\left(\int_{0}^{r_{n}}\left|\sum_{k=n+1}^{\infty} f_{k}(z t)\right|^{p} d t\right)^{1 / q} \\
& >\left(G(z)-2^{-(m+n)}\right)^{1 / q}-\left(\sum_{k=n+1}^{\infty} 2^{-k}\right)^{p / q} \\
& =\left(G(z)-2^{-(m+n)}\right)^{1 / q}-2^{-n p / q} \underset{n \rightarrow \infty}{\longrightarrow}(G(z))^{1 / q}
\end{aligned}
$$

Therefore,

$$
\mathcal{R}^{p}(f)(z) \geq G(z) \text { for all } z \in \partial \mathbb{D} \text {. }
$$


These inequalities establish the conclusion $\mathcal{R}^{p}(f)=G$ on $\partial \mathbb{D}$. Finally, it follows from Theorem 2 that $\mathcal{R}^{p}(f) \in \mathcal{C}(\overline{\mathbb{D}})$.

\section{Divergent Taylor Series}

Lemma 7 Let $h \in \mathcal{C}(\partial \mathbb{D})$ be a strictly positive function, $\phi \in \mathcal{C}(\overline{\mathbb{D}})$ be a bounded function and $a, \theta, \varepsilon \in(0,1)$. There exist a natural number $N \in \mathbb{N}$ and a sequence of orthogonal polynomials $\left\{P_{n}\right\}_{n \in \mathbb{N}}$ such that on $\partial \mathbb{D}$ the following statements hold

(s1) $\mathcal{R}^{2}\left(P_{k}\right)<a h, k=0,1, \ldots$

(s2) $\mathcal{R}^{2}\left(\sum_{j=0}^{k} P_{j}\right)<h, k=0,1, \ldots$

(s3) $\mathcal{R}^{2}\left(\sum_{j=0}^{k} P_{j}\right)>\frac{1}{2} a\left(h-\mathcal{R}^{2}\left(\sum_{j=0}^{k-1} P_{j}\right)\right)+\mathcal{R}^{2}\left(\sum_{j=0}^{k-1} P_{j}\right), k=1,2, \ldots$

$$
\mid \begin{aligned}
& \mathcal{R}^{2}\left(\sum_{j=0}^{k} P_{j}\right)-\sum_{j=0}^{k} \mathcal{R}^{2}\left(P_{j}\right) \mid<\varepsilon, k=1,2, \ldots \\
& \mathcal{R}^{2}\left(\phi+\sum_{j=0}^{k} P_{j}\right)-\sum_{j=0}^{k} \mathcal{R}^{2}\left(P_{j}\right)-\mathcal{R}^{2}(\phi) \mid<\varepsilon, k=1,2, \ldots
\end{aligned}
$$

(s6) $\mathcal{R}^{2}\left(\sum_{j=0}^{N} P_{j}\right)>\theta h$.

Proof First notice that $P_{0} \equiv 0$ satisfies conditions $(s 1)-(s 2)$. Then Lemmas 3 and 5 applied to

$$
H:=\frac{5}{6} a h, \quad g:=\phi, \quad \varepsilon_{2}:=\frac{1}{5}, \quad \varepsilon_{3}:=\varepsilon
$$

produces a polynomial $P_{1}$ with the following properties

- $\frac{1}{2} a h<\frac{4}{5} H<\mathcal{R}^{2}\left(P_{1}\right)<H<a h$ on $\partial \mathbb{D}$

- $\mathcal{R}^{2}\left(P_{1}\right)+\mathcal{R}^{2}(\phi)-\varepsilon<\mathcal{R}^{2}\left(\phi+P_{1}\right)<\mathcal{R}^{2}\left(P_{1}\right)+\mathcal{R}^{2}(\phi)-\varepsilon$ on $\partial \mathbb{D}$.

Of course, the above statements imply $(s 1)-(s 5)$ immediately.

We then proceed inductively as follows: Suppose that orthogonal polynomials $P_{0}, P_{1}, \ldots, P_{k}$ have been found such that conditions $(s 1)-(s 5)$ hold. By $(s 4)$ and (s5), there exist $\rho_{1}, \rho_{2} \in(0,1)$ such that

$$
\text { - } \mid \begin{aligned}
& \mathcal{R}^{2}\left(\sum_{j=0}^{k} P_{j}\right)-\sum_{j=0}^{k} \mathcal{R}^{2}\left(P_{j}\right) \mid<\rho_{1}<\varepsilon \text { on } \partial \mathbb{D} \\
& \text { - }\left|\mathcal{R}^{2}\left(\phi+\sum_{j=0}^{k} P_{j}\right)-\sum_{j=0}^{k} \mathcal{R}^{2}\left(P_{j}\right)-\mathcal{R}^{2}(\phi)\right|<\rho_{2}<\varepsilon \text { on } \partial \mathbb{D} .
\end{aligned}
$$


Select $N_{1} \in \mathbb{N}$ such that $N_{1}>\operatorname{deg}\left(P_{k}\right)$. For our inductive step, again we may apply Lemmas 3 and 5 to

$$
\begin{aligned}
H:= & \frac{5}{6} a\left(h-\mathcal{R}^{2}\left(\sum_{j=0}^{k} P_{j}\right)\right), \quad g_{1}:=\sum_{j=0}^{k} P_{j}, \quad g_{2}:=\sum_{j=0}^{k} P_{j}+\phi, \quad \varepsilon_{2}:=\frac{1}{5}, \\
& \varepsilon_{3}:=\min \left\{\frac{1}{6} \inf _{z \in \partial \mathbb{D}} a\left(h(z)-\mathcal{R}^{2}\left(\sum_{j=0}^{k} P_{j}\right)(z)\right), \varepsilon-\rho_{1}, \varepsilon-\rho_{2}\right\}
\end{aligned}
$$

to obtain a polynomial $P_{k+1}(z):=\left(2 n_{k+1}+1\right)^{1 / 2} Q_{k+1}(z) z^{n_{k+1}}$, where $n_{k+1} \geq N_{1}$ and $Q_{k+1} \neq 0$ on $\partial \mathbb{D}$. Therefore, the polynomial $P_{k+1}$ is orthogonal to $P_{0}, P_{1}, \ldots, P_{k}$ and satisfies the following statements

(a) $\left(1-\varepsilon_{2}\right) H<\mathcal{R}^{2}\left(P_{k+1}\right)<H$ on $\partial \mathbb{D}$

(b) $\mathcal{R}^{2}\left(g_{1}\right)+\mathcal{R}^{2}\left(P_{k+1}\right)-\varepsilon_{3}<\mathcal{R}^{2}\left(g_{1}+P_{k+1}\right)<\mathcal{R}^{2}\left(g_{1}\right)+\mathcal{R}^{2}\left(P_{k+1}\right)+\varepsilon_{3}$ on $\partial \mathbb{D}$

(c) $\mathcal{R}^{2}\left(g_{2}\right)+\mathcal{R}^{2}\left(P_{k+1}\right)-\varepsilon_{3}<\mathcal{R}^{2}\left(g_{2}+P_{k+1}\right)<\mathcal{R}^{2}\left(g_{2}\right)+\mathcal{R}^{2}\left(P_{k+1}\right)+\varepsilon_{3}$ on $\partial \mathbb{D}$.

Direct estimation on $\partial \mathbb{D}$ gives $(s 2)$

$$
\begin{aligned}
\mathcal{R}^{2}\left(\sum_{j=0}^{k+1} P_{j}\right)< & \mathcal{R}^{2}\left(\sum_{j=0}^{k} P_{j}\right)+\mathcal{R}^{2}\left(P_{k+1}\right)+\varepsilon_{3} \\
< & \mathcal{R}^{2}\left(\sum_{j=0}^{k} P_{j}\right)+\frac{5}{6} a\left(h-\mathcal{R}^{2}\left(\sum_{j=0}^{k} P_{j}\right)\right) \\
& +\frac{1}{6} a\left(h-\mathcal{R}^{2}\left(\sum_{j=0}^{k} P_{j}\right)\right) \\
\leq & h
\end{aligned}
$$

and (s3)

$$
\begin{aligned}
\mathcal{R}^{2}\left(\sum_{j=0}^{k+1} P_{j}\right)> & \mathcal{R}^{2}\left(\sum_{j=0}^{k} P_{j}\right)+\mathcal{R}^{2}\left(P_{k+1}\right)-\varepsilon_{3} \\
> & \mathcal{R}^{2}\left(\sum_{j=0}^{k} P_{j}\right)+\frac{2}{3} a\left(h-\mathcal{R}^{2}\left(\sum_{j=0}^{k} P_{j}\right)\right) \\
& -\frac{1}{6} a\left(h-\mathcal{R}^{2}\left(\sum_{j=0}^{k} P_{j}\right)\right) \\
\geq & \mathcal{R}^{2}\left(\sum_{j=0}^{k} P_{j}\right)+\frac{1}{2} a\left(h-\mathcal{R}^{2}\left(\sum_{j=0}^{k} P_{j}\right)\right) .
\end{aligned}
$$


Furthermore, combining (a)-(c) and the induction hypothesis gives conditions $(s 4)-$ (s5)

$$
\begin{aligned}
\mathcal{R}^{2}\left(\sum_{j=0}^{k+1} P_{j}\right) & <\mathcal{R}^{2}\left(\sum_{j=0}^{k} P_{j}\right)+\mathcal{R}^{2}\left(P_{k+1}\right)+\varepsilon_{3}<\sum_{j=0}^{k+1} \mathcal{R}^{2}\left(P_{j}\right)+\rho_{1}+\varepsilon_{3} \\
& =\sum_{j=0}^{k+1} \mathcal{R}^{2}\left(P_{j}\right)+\varepsilon
\end{aligned}
$$

and

$$
\begin{aligned}
\mathcal{R}^{2}\left(\sum_{j=0}^{k+1} P_{j}+\phi\right)< & \mathcal{R}^{2}\left(\sum_{j=0}^{k} P_{j}+\phi\right) \\
& +\mathcal{R}^{2}\left(P_{k+1}\right)+\varepsilon_{3}<\mathcal{R}^{2}(\phi)+\sum_{j=0}^{k+1} \mathcal{R}^{2}\left(P_{j}\right)+\rho_{2}+\varepsilon_{3} \\
< & \mathcal{R}^{2}(\phi)+\mathcal{R}^{2}\left(\sum_{j=0}^{k+1} P_{j}\right)+\varepsilon .
\end{aligned}
$$

Similarly,

$$
\mathcal{R}^{2}\left(\sum_{j=0}^{k+1} P_{j}\right)>\sum_{j=0}^{k+1} \mathcal{R}^{2}\left(P_{j}\right)-\varepsilon
$$

and

$$
\mathcal{R}^{2}\left(\sum_{j=0}^{k+1} P_{j}+\phi\right)>\mathcal{R}^{2}(\phi)+\mathcal{R}^{2}\left(\sum_{j=0}^{k+1} P_{j}\right)-\varepsilon
$$

The induction is now complete.

Observe that statements $(s 2)$ and (s3) imply that

$$
\lim _{n \rightarrow \infty} \mathcal{R}^{2}\left(\sum_{j=0}^{n} P_{j}\right)(z)=h(z), \quad z \in \partial \mathbb{D}
$$

and

$$
\left\{\mathcal{R}^{2}\left(\sum_{j=0}^{n} P_{j}\right)\right\}_{n \in \mathbb{N}}
$$


is an increasing sequence. Hence, by Dini's theorem, $\mathcal{R}^{2}\left(\sum_{j=0}^{n} P_{j}\right)$ converges uniformly to $h$. Therefore, there exists $N \in \mathbb{N}$ such that

$$
\mathcal{R}^{2}\left(\sum_{j=0}^{N} P_{j}\right)>\theta h \quad \text { on } \partial \mathbb{D} \text {. }
$$

Theorem 8 There exists a holomorphic function $f(z)=\sum_{n=0}^{\infty} a_{n} z^{n}$ such that

$$
\begin{aligned}
& \text { 1. } \mathcal{R}^{2}(f) \in \mathcal{C}(\overline{\mathbb{D}}) \\
& \text { 2. } \sum_{n=1}^{\infty}\left(\frac{\left|a_{n}\right|^{2}}{2 n+1}\right)^{s}=\infty, \quad s \in[0,1) \text {. }
\end{aligned}
$$

Proof For every $j \in \mathbb{N}$ we may apply the preceding lemma with $\phi \equiv 0, h \equiv$ $1, a:=2^{-j}, \theta:=1 / 2$ to obtain $N_{j} \in \mathbb{N}$ and a sequence of orthogonal polynomials $\left\{P_{j k}\right\}_{0 \leq k \leq N_{j}}, j \in \mathbb{N}$ such that

(a) $\mathcal{R}^{2}\left(P_{j k}\right)<2^{-j}, \quad k=0, \ldots, N_{j}$

(b) $\frac{1}{2}<\mathcal{R}^{2}\left(\sum_{k=0}^{N_{j}} P_{j k}\right)<1$.

Observe that if $n>m$, then

$$
\begin{aligned}
& \sup _{z \in \partial \mathbb{D}}\left(\mathcal{R}^{2}\left(\sum_{j=1}^{n} \frac{1}{j^{2}} \sum_{k=0}^{N_{j}} P_{j k}-\sum_{j=1}^{m} \frac{1}{j^{2}} \sum_{k=0}^{N_{j}} P_{j k}\right)(z)\right)^{1 / 2} \\
& =\sup _{z \in \partial \mathbb{D}}\left(\mathcal{R}^{2}\left(\sum_{j=m+1}^{n} \frac{1}{j^{2}} \sum_{k=0}^{N_{j}} P_{j k}\right)(z)\right)^{1 / 2} \\
& \leq \sup _{z \in \partial \mathbb{D}}\left(\sum_{j=m+1}^{n} \frac{1}{j^{2}}\left(\mathcal{R}^{2}\left(\sum_{k=0}^{N_{j}} P_{j k}\right)(z)\right)^{1 / 2}\right) \\
& \leq \sum_{j=m+1}^{n} \frac{1}{j^{2}} \underset{\substack{n, m \rightarrow \infty \\
\rightarrow m}}{ } 0 .
\end{aligned}
$$

It follows from the above that

$$
\left\{\sum_{j=1}^{n} \frac{1}{j^{2}} \sum_{k=0}^{N_{j}} P_{j k}\right\}_{n \in \mathbb{N}}
$$


is a Cauchy sequence in $\mathcal{H} \mathcal{R}^{2}(\mathbb{D})$ which by Proposition 1 is convergent in $\mathcal{H} \mathcal{R}^{2}(\mathbb{D})$.

Therefore, we may define a function

$$
f:=\sum_{j=1}^{\infty} \frac{1}{j^{2}} \sum_{k=0}^{N_{j}} P_{j k} \in \mathcal{O}(\mathbb{D}) .
$$

Denote $\mathcal{R}^{2}\left(\sum_{j=1}^{n} 1 / j^{2} \sum_{k=0}^{N_{j}} P_{j k}\right)$ by $\mathcal{P}_{n}$, for the moment. If $n>m$, then on $\partial \mathbb{D}$

$$
\begin{aligned}
& \left|\left(\mathcal{P}_{n}\right)^{1 / 2}-\left(\mathcal{P}_{m}\right)^{1 / 2}\right| \\
& =\left|\left(\mathcal{R}^{2}\left(\sum_{j=1}^{n} \frac{1}{j^{2}} \sum_{k=0}^{N_{j}} P_{j k}\right)\right)^{1 / 2}-\left(\mathcal{R}^{2}\left(\sum_{j=1}^{m} \frac{1}{j^{2}} \sum_{k=0}^{N_{j}} P_{j k}\right)\right)^{1 / 2}\right| \\
& \leq\left(\mathcal{R}^{2}\left(\sum_{j=m+1}^{n} \frac{1}{j^{2}} \sum_{k=0}^{N_{j}} P_{j k}\right)\right)^{1 / 2} \leq \sum_{j=m+1}^{n}\left(\mathcal{R}^{2}\left(\frac{1}{j^{2}} \sum_{k=0}^{N_{j}} P_{j k}\right)\right)^{1 / 2} \\
& =\sum_{j=m+1}^{n} \frac{1}{j^{2}}\left(\mathcal{R}^{2}\left(\sum_{k=0}^{N_{j}} P_{j k}\right)\right)^{1 / 2} \leq \sum_{j=m+1}^{n} \frac{1}{j^{2}} \underset{n, m \rightarrow \infty}{\longrightarrow} 0 .
\end{aligned}
$$

Observe that there exists a constant $C>0$ such that $\sup _{z \in \partial \mathbb{D}}\left|\mathcal{P}_{n}(z)\right|<C$ for all $n \in \mathbb{N}$. Hence,

$$
\begin{aligned}
\sup _{z \in \partial \mathbb{D}}\left|\mathcal{P}_{n}(z)-\mathcal{P}_{m}(z)\right| & =\sup _{z \in \partial \mathbb{D}}\left|\left(\left(\mathcal{P}_{n}(z)\right)^{1 / 2}+\left(\mathcal{P}_{m}(z)\right)^{1 / 2}\right)\left(\left(\mathcal{P}_{n}(z)\right)^{1 / 2}-\left(\mathcal{P}_{m}(z)\right)^{1 / 2}\right)\right| \\
& \leq 2 C \sup _{z \in \partial \mathbb{D}}\left|\left(\mathcal{P}_{n}(z)\right)^{1 / 2}-\left(\mathcal{P}_{m}(z)\right)^{1 / 2}\right| \underset{n, m \rightarrow \infty}{\longrightarrow} 0 .
\end{aligned}
$$

This implies that

$$
\left\{\mathcal{R}^{2}\left(\sum_{j=1}^{n} \frac{1}{j^{2}} \sum_{k=0}^{N_{j}} P_{j k}\right)\right\}_{n \in \mathbb{N}}
$$

is a Cauchy sequence in the space of continuous functions, so $\mathcal{R}^{2}(f) \in \mathcal{C}(\partial \mathbb{D})$ and consequently by Theorem 2 , the function $\mathcal{R}^{2}(f)$ is also continuous in $\overline{\mathbb{D}}$.

Now we turn to conclusion 2. Let $P_{j k}(z)=\sum_{n \in I_{j k}} p_{j k n}(z)=\sum_{n \in I_{j k}} a_{j k n} z^{n}$ be the homogeneous expansion of $P_{j k}$, where $I_{j k}$ is the set of monomials' degrees of the polynomial $P_{j k}$. By construction, $I_{j m} \cap I_{i k}=\emptyset$ for $(j, m) \neq(i, k)$. Set $s \in[0,1)$. Notice that by statement b),

$$
\left\|P_{j k}\right\|_{2, \mathbb{D}}^{2}=\left\|\mathcal{R}^{2}\left(P_{j k}\right)\right\|_{1, \partial \mathbb{D}}<2^{-j}, \quad k=0, \ldots, N_{j},
$$

which implies that

$$
\left\|P_{j k}\right\|_{2, \mathbb{D}}^{2 s} \geq\left\|P_{j k}\right\|_{2, \mathbb{D}}^{2 s}\left(\left\|P_{j k}\right\|_{2, \mathbb{D}}^{2} 2^{j}\right)^{1-s}=\left\|P_{j k}\right\|_{2, \mathbb{D}}^{2} 2^{j(1-s)}, \quad k=0, \ldots, N_{j}
$$


Due to the orthogonality of the polynomials $p_{j k n}$, a simple calculation shows that

$$
\begin{aligned}
\left\|P_{j k}\right\|_{2, \mathbb{D}}^{2} & =\left\|\sum_{n \in I_{j k}} p_{j k n}\right\|_{2, \mathbb{D}}^{2}=\sum_{n \in I_{j k}}\left\|p_{j k n}\right\|_{2, \mathbb{D}}^{2}=\sum_{n \in I_{j k}} \int_{\partial \mathbb{D}} \int_{0}^{1}\left|a_{j k n} z^{n} t^{n}\right|^{2} d t d \sigma(z) \\
& =\sum_{n \in I_{j k}} \int_{0}^{1}\left|a_{j k n}\right|^{2} t^{2 n} d t=\sum_{n \in I_{j k}} \frac{\left|a_{j k n}\right|^{2}}{2 n+1}
\end{aligned}
$$

Finally, we conclude from all this that

$$
\begin{aligned}
\sum_{j, k, n}\left(\frac{1}{j^{4}} \frac{\left|a_{j k n}\right|^{2}}{2 n+1}\right)^{s} & \geq \sum_{j, k}\left(\frac{1}{j^{4}}\right)^{s}\left(\sum_{n \in I_{j k}} \frac{\left|a_{j k n}\right|^{2}}{2 n+1}\right)^{s}=\sum_{j, k} \frac{1}{j^{4 s}}\left\|P_{j k}\right\|_{2, \mathbb{D}}^{2 s} \\
& =\sum_{j=1}^{\infty} \frac{1}{j^{4 s}} \sum_{k=0}^{N_{j}}\left\|P_{j k}\right\|_{2, \mathbb{D}}^{2 s} \geq \sum_{j=1}^{\infty} \frac{1}{j^{4 s}} \sum_{k=0}^{N_{j}}\left\|P_{j k}\right\|_{2, \mathbb{D}}^{2} 2^{j(1-s)} \\
& =\sum_{j=1}^{\infty} \frac{1}{j^{4 s}}\left\|\sum_{k=0}^{N_{j}} P_{j k}\right\|_{2, \mathbb{D}}^{2} 2^{j(1-s)}>\sum_{j=1}^{\infty} \frac{1}{2 j^{4 s}} 2^{j(1-s)} \\
& =\infty
\end{aligned}
$$

for $s \in[0,1)$. The proof of the theorem is now complete.

Observe that the above theorem does not depend on all the statements from Lemma 7. However, we may improve Theorem 8 so that the function $f$ satisfies also the Radon inversion problem. This can be done by applying the full version of Lemma 7 with suitable arguments.

Theorem 9 Let $\Phi \in \mathcal{C}(\partial \mathbb{D})$ be a strictly positive function. There exists a holomorphic function $f(z)=\sum_{n=0}^{\infty} a_{n} z^{n}$ such that

1. $\mathcal{R}^{2}(f)=\Phi$ on $\partial \mathbb{D}$

2. $\mathcal{R}^{2}(f) \in \mathcal{C}(\overline{\mathbb{D}})$

3. $\sum_{n=0}^{\infty}\left(\frac{\left|a_{n}\right|^{2}}{2 n+1}\right)^{s}=\infty, \quad s \in[0,1)$.

Proof Without loss of generality let us assume that $\sup _{z \in \partial \mathbb{D}}|\Phi(z)|<1$. We shall construct a sequence of orthogonal polynomials $\left\{Q_{k}\right\}_{k=0}^{\infty}$ with the following properties

( $p 1)$ each $Q_{k}=\sum_{j=0}^{N_{k}} P_{k j}$ is a sum of orthogonal polynomials $P_{k j}$

(p2) $\mathcal{R}^{2}\left(P_{k j}\right)<\left\|\Phi-\mathcal{R}^{2}\left(\sum_{m=0}^{k-1} Q_{m}\right)\right\|_{1, \partial \mathbb{D}}^{k} \quad$ on $\partial \mathbb{D}, \quad j=0,1, \ldots, N_{k}$ 
(p3) $\mathcal{R}^{2}\left(\sum_{m=0}^{k} Q_{m}\right)<\Phi$ on $\partial \mathbb{D}$

(p4) $\mathcal{R}^{2}\left(\sum_{m=0}^{k} Q_{m}\right)>\frac{1}{4}\left(\Phi-\mathcal{R}^{2}\left(\sum_{m=0}^{k-1} Q_{m}\right)\right)+\mathcal{R}^{2}\left(\sum_{m=0}^{k-1} Q_{m}\right)$ on $\partial \mathbb{D}$

(p5) $\left\|Q_{k}\right\|_{2, \mathbb{D}}^{2}>\frac{1}{2}\left\|\Phi-\mathcal{R}^{2}\left(\sum_{m=0}^{k-1} Q_{m}\right)\right\|_{1, \partial \mathbb{D}}$

We will apply Lemma 7 iteratively, with new $a, h, \phi, \theta, \varepsilon$ at each iteration. Let us begin our process by selecting $Q_{0} \equiv 0$. Next apply Lemma 7 to

$$
a:=\inf _{z \in \partial \mathbb{D}}|\Phi(z)|, \quad h:=\Phi, \quad \phi \equiv 0, \quad \theta:=\frac{1}{2} .
$$

This produces $N_{1} \in \mathbb{N}$ and orthogonal polynomials $P_{10}, P_{11}, \ldots, P_{1 N_{1}}$ such that

- $\mathcal{R}^{2}\left(P_{1 j}\right)<\|\Phi\|_{1, \partial \mathbb{D}}$ on $\partial \mathbb{D}, \quad j=0,1, \ldots, N_{1}$

- $\mathcal{R}^{2}\left(\sum_{j=0}^{N_{1}} P_{1 j}\right)<\Phi$ on $\partial \mathbb{D}$

- $\mathcal{R}^{2}\left(\sum_{j=0}^{N_{1}} P_{1 j}\right)>\frac{1}{2} \Phi>\frac{1}{4} \Phi$ on $\partial \mathbb{D}$.

Now it is enough to define $Q_{1}:=\sum_{j=0}^{N_{1}} P_{1 j}$. Proceeding inductively, let us assume that orthogonal polynomials $Q_{0}, Q_{1}, \ldots, Q_{k}$ with the required properties $(p 1)-(p 5)$ have been constructed. We then may apply Lemma 7 with

$$
\begin{aligned}
& a:=\left\|\Phi-\mathcal{R}^{2}\left(\sum_{m=0}^{k} Q_{m}\right)\right\|_{1, \partial \mathbb{D}}^{k+1}, \quad h:=\frac{3}{4}\left(\Phi-\mathcal{R}^{2}\left(\sum_{m=0}^{k} Q_{m}\right)\right), \\
& \phi:=\sum_{m=0}^{k} Q_{m}, \quad \theta:=\frac{2}{3}, \quad \varepsilon:=\frac{1}{8} \inf _{z \in \partial \mathbb{D}}\left(\Phi(z)-\mathcal{R}^{2}\left(\sum_{m=0}^{k} Q_{m}\right)(z)\right)
\end{aligned}
$$

to obtain $N_{k+1} \in \mathbb{N}$ and orthogonal polynomials $P_{k+1,0}, P_{k+1,1}, \ldots, P_{k+1, N_{k+1}}$ that are also orthogonal to the polynomials $Q_{0}, Q_{1}, \ldots, Q_{k}$ and satisfy the following conditions

(a) $\mathcal{R}^{2}\left(P_{k+1, j}\right)<\left\|\Phi-\mathcal{R}^{2}\left(\sum_{m=0}^{k} Q_{m}\right)\right\|_{1, \partial \mathbb{D}}^{k+1}$ on $\partial \mathbb{D}, \quad j=0, \ldots, N_{k+1}$

(b) $\mathcal{R}^{2}\left(\sum_{j=0}^{N_{k+1}} P_{k+1, j}\right)<h=\frac{3}{4}\left(\Phi-\mathcal{R}^{2}\left(\sum_{m=0}^{k} Q_{m}\right)\right)$ on $\partial \mathbb{D}$

(c) $\mathcal{R}^{2}\left(\sum_{j=0}^{N_{k+1}} P_{k+1, j}\right)>\theta h=\frac{1}{2}\left(\Phi-\mathcal{R}^{2}\left(\sum_{m=0}^{k} Q_{m}\right)\right)$ on $\partial \mathbb{D}$ 
(d) $\left|\mathcal{R}^{2}\left(\sum_{m=0}^{k} Q_{m}+\sum_{j=0}^{N_{k+1}} P_{k+1, j}\right)-\mathcal{R}^{2}\left(\sum_{m=0}^{k} Q_{m}\right)-\mathcal{R}^{2}\left(\sum_{j=0}^{N_{k+1}} P_{k+1, j}\right)\right|<2 \varepsilon$ on $\partial \mathbb{D}$.

If we define $Q_{k+1}:=\sum_{j=0}^{N_{k+1}} P_{k+1, j}$, then statements $(p 1)-(p 2)$ and $(p 5)$ immediately hold. Other properties $(p 3)-(p 4)$ follow from simple calculations

$$
\begin{aligned}
\mathcal{R}^{2}\left(\sum_{m=0}^{k+1} Q_{m}\right)< & \mathcal{R}^{2}\left(\sum_{m=0}^{k} Q_{m}\right)+\mathcal{R}^{2}\left(Q_{k+1}\right)+2 \varepsilon<\mathcal{R}^{2}\left(\sum_{m=0}^{k} Q_{m}\right) \\
& +\Phi-\mathcal{R}^{2}\left(\sum_{m=0}^{k} Q_{m}\right)=\Phi
\end{aligned}
$$

and

$$
\begin{aligned}
\mathcal{R}^{2}\left(\sum_{m=0}^{k+1} Q_{m}\right)> & \mathcal{R}^{2}\left(\sum_{m=0}^{k} Q_{m}\right)+\mathcal{R}^{2}\left(Q_{k+1}\right)-2 \varepsilon \geq \mathcal{R}^{2}\left(\sum_{m=0}^{k} Q_{m}\right) \\
& +\frac{1}{4}\left(\Phi-\mathcal{R}^{2}\left(\sum_{m=0}^{k} Q_{m}\right)\right) .
\end{aligned}
$$

That completes the induction. Combining statements $(p 3)-(p 4)$ iteratively gives the following

$$
\begin{aligned}
\Phi-\mathcal{R}^{2}\left(\sum_{m=0}^{k} Q_{m}\right) & <\Phi-\frac{1}{4}\left(\Phi-\mathcal{R}^{2}\left(\sum_{m=0}^{k-1} Q_{m}\right)\right)-\mathcal{R}^{2}\left(\sum_{m=0}^{k-1} Q_{m}\right) \\
& =\frac{3}{4}\left(\Phi-\mathcal{R}^{2}\left(\sum_{m=0}^{k-1} Q_{m}\right)\right) \\
& <\left(\frac{3}{4}\right)^{k} \Phi .
\end{aligned}
$$

This leads to the conclusion that $\lim _{n \rightarrow \infty} \mathcal{R}^{2}\left(\sum_{k=0}^{n} Q_{k}\right)=\Phi$ on $\partial \mathbb{D}$. Moreover, if $n>m$, then

$$
\begin{aligned}
\sup _{z \in \partial \mathbb{D}}\left(\mathcal{R}^{2}\left(\sum_{k=0}^{n} Q_{k}-\sum_{k=0}^{m} Q_{j k}\right)(z)\right)^{1 / 2} & =\sup _{z \in \partial \mathbb{D}}\left(\mathcal{R}^{2}\left(\sum_{k=m+1}^{n} Q_{k}\right)(z)\right)^{1 / 2} \\
& \leq \sup _{z \in \partial \mathbb{D}}\left(\sum_{k=m+1}^{n}\left(\mathcal{R}^{2}\left(Q_{k}\right)(z)\right)^{1 / 2}\right) \\
& \leq \sup _{z \in \partial \mathbb{D}}\left(\sum_{k=m+1}^{n} \frac{\sqrt{3}}{2}\left(\Phi-\mathcal{R}^{2}\left(\sum_{m=0}^{k-1} Q_{m}\right)\right)^{1 / 2}\right) \\
& <\sum_{j=m+1}^{n}\left(\frac{\sqrt{3}}{2}\right)^{k}\|\Phi\|^{1 / 2} \\
& \underset{n, m \rightarrow \infty}{\longrightarrow} 0 .
\end{aligned}
$$


Hence, $\left\{\sum_{k=0}^{n} Q_{k}\right\}_{n \in \mathbb{N}}$ is a Cauchy sequence in $\mathcal{H} \mathcal{R}^{2}(\mathbb{D})$ and by Proposition 1 is convergent in $\mathcal{H} \mathcal{R}^{2}(\mathbb{D})$. It follows that the function $f:=\sum_{k=0}^{\infty} Q_{k}$ is well-defined and holomorphic in $\mathbb{D}$. Then Proposition 1 also tells us that $\mathcal{R}^{2}(f)=\lim _{n \rightarrow \infty} \mathcal{R}^{2}\left(\sum_{k=0}^{n} Q_{k}\right)=\Phi$ on $\partial \mathbb{D}$. Furthermore, due to Theorem 2 the function $\mathcal{R}^{2}(f)$ is continuous in $\overline{\mathbb{D}}$.

Now we prove the second part of the theorem. Taking into account that $Q_{0} \equiv 0$, we may observe that $f=\sum_{k=1}^{\infty} Q_{k}=\sum_{k=1}^{\infty} \sum_{j=0}^{N_{k}} P_{k j}$. Property ( $p 2$ ) implies that

$$
\left\|P_{k j}\right\|_{2, \mathbb{D}}^{2}=\left\|\mathcal{R}^{2}\left(P_{k j}\right)\right\|_{1, \partial \mathbb{D}}<\left\|\Phi-\mathcal{R}^{2}\left(\sum_{m=0}^{k-1} Q_{m}\right)\right\|_{1, \partial \mathbb{D}}^{k}, \quad j=0, \ldots, N_{k},
$$

which is equivalent to

$$
\left\|\Phi-\mathcal{R}^{2}\left(\sum_{m=0}^{k-1} Q_{m}\right)\right\|_{1, \partial \mathbb{D}}^{-k}\left\|P_{k j}\right\|_{2, \mathbb{D}}^{2}<1, \quad j=0, \ldots, N_{k} .
$$

If $s \in[0,1)$, then we may apply above inequality to obtain that

$$
\begin{aligned}
\left\|P_{k j}\right\|_{2, \mathbb{D}}^{2 s} & >\left\|P_{k j}\right\|_{2, \mathbb{D}}^{2 s}\left(\left\|\Phi-\mathcal{R}^{2}\left(\sum_{m=0}^{k-1} Q_{m}\right)\right\|_{1, \partial \mathbb{D}}^{-k}\left\|P_{k j}\right\|_{2, \mathbb{D}}^{2}\right)^{1-s}, j=0, \ldots, N_{k} . \\
& =\left\|\Phi-\mathcal{R}^{2}\left(\sum_{m=0}^{k-1} Q_{m}\right)\right\|_{1, \partial \mathbb{D}}^{k(s-1)}\left\|P_{k j}\right\|_{2, \mathbb{D}}^{2}
\end{aligned}
$$

Let

$$
P_{k j}(z)=\sum_{n \in I_{k j}} p_{k j n}(z)=\sum_{n \in I_{k j}} a_{k j n} z^{n}
$$

be the homogenous expansion of $P_{k j}$, where $I_{k j}$ is the set of monomial's degrees of the polynomial $P_{k j}$. By construction, $I_{m j} \cap I_{k i}=\emptyset$ for $(m, j) \neq(k, i)$. Orthogonality of the polynomials $p_{k j n}$ gives the equality

$$
\left\|P_{k j}\right\|_{2, \mathbb{D}}^{2}=\sum_{n \in I_{k j}}\left\|p_{k j n}\right\|_{2, \mathbb{D}}^{2}=\sum_{n \in I_{k j}} \int_{\partial \mathbb{D}} \int_{0}^{1}\left|a_{k j n} z^{n} t^{n}\right|^{2} d t d \sigma(z)=\sum_{n \in I_{k j}} \frac{\left|a_{k j n}\right|^{2}}{2 n+1}
$$

We conclude with the following estimation

$$
\begin{aligned}
\sum_{k, j, n}\left(\frac{\left|a_{k j n}\right|^{2}}{2 n+1}\right)^{s} & \geq \sum_{k, j}\left(\sum_{n \in I_{k j}} \frac{\left|a_{k j n}\right|^{2}}{2 n+1}\right)^{s}=\sum_{k=1}^{\infty} \sum_{j=0}^{N_{k}}\left\|P_{k j}\right\|_{2, \mathbb{D}}^{2 s} \\
& \stackrel{(9)}{>} \sum_{k=1}^{\infty}\left\|\Phi-\mathcal{R}^{2}\left(\sum_{m=0}^{k-1} Q_{m}\right)\right\|_{1, \mathfrak{D}}^{k(s-1)} \sum_{j=0}^{N_{k}}\left\|P_{k j}\right\|_{2, \mathbb{D}}^{2}
\end{aligned}
$$




$$
\begin{aligned}
& =\sum_{k=1}^{\infty}\left\|\Phi-\mathcal{R}^{2}\left(\sum_{m=0}^{k-1} Q_{m}\right)\right\|_{1, \partial \mathbb{D}}^{k(s-1)}\left\|Q_{k}\right\|_{2, \mathbb{D}}^{2} \\
& \stackrel{(\text { p } 5)}{>} \sum_{k=1}^{\infty} \frac{1}{2}\left\|\Phi-\mathcal{R}^{2}\left(\sum_{m=0}^{k-1} Q_{m}\right)\right\|_{1, \partial \mathbb{D}}^{k(s-1)+1} \\
& \geq \sum_{k>1 /(1-s)} \frac{1}{2}=\infty
\end{aligned}
$$

for $s \in[0,1)$. This completes the proof.

It is worth mentioning that in contrast to Theorem 6, in the above theorem we have applied Proposition 1 to show that the function that has been constructed in Theorem 9 satisfies the Radon inversion problem. This provides two different approaches in proving solvability of the problem under consideration.

\section{Final Remarks}

Herein, we shall give some remarks about the comparison of the Radon inversion problem we have solved in this paper and the generalization of the inner function problem formulated in (2). The second problem in one variable can be easily solved by applying the Schwarz integral. There exists a continuous real valued function $\phi$ such that $G=e^{\phi}$. Solving a standard Dirichlet problem for $\phi$ we obtain a harmonic function $u_{\phi} \in \mathcal{C}(\overline{\mathbb{D}})$ such that $u_{\phi}=\phi$ on $\partial \mathbb{D}$. Then, the Schwarz integral for $u_{\phi}$ produces a function $\mathcal{S}\left(u_{\phi}\right) \in \mathcal{O}(\mathbb{D})$ such that $\operatorname{Re}\left(\mathcal{S}\left(u_{\phi}\right)\right) \in \mathcal{C}(\overline{\mathbb{D}})$ and $\operatorname{Re}\left(\mathcal{S}\left(u_{\phi}\right)\right)=$ $\phi$ on $\partial \mathbb{D}$ (see e.g. [3,7]). Now it is enough to take $g:=e^{\mathcal{S}\left(u_{\phi}\right)}$. Here we observe that if $f, g \in \mathcal{O}(\mathbb{D})$ are solutions of (1) and (2) respectively, then both functions $|g|$ and $\mathcal{R}^{p}(f)$ are continuous up to the boundary $\partial \mathbb{D}$ as has been shown in Theorem 2.

However, there is a significant difference between the problems under consideration regarding zero sets of their possible solutions. On the one hand, notice that if $g$ is a solution of the generalized inner function problem (2), then continuity of $|g|$ up to the boundary $\partial \mathbb{D}$ implies that $g$ cannot have infinitely many zeros. On the other hand, there exists a solution of the Radon inversion problem that has infinitely many zeros. In Theorem 6 we have constructed a function $f(z):=\sum_{k=1}^{\infty}\left(n_{k} p+1\right)^{1 / p} Q_{k}(z) z^{n_{k}}$, where $Q_{k}$ is a polynomial with certain properties. The function $f$ is a solution to (1). Observe that it may be modified suitably to have infinitely many zeros in the following way: For $m \in \mathbb{N}$ there exist $R_{m} \in(0,1)$ and $n_{m} \in \mathbb{N}$ sufficiently large such that

$$
\left|\sum_{k=1}^{m-1}\left(n_{k} p+1\right)^{1 / p} Q_{k}(z) z^{n_{k}}\right|<\left(n_{m} p+1\right)^{1 / p} R_{m}^{n_{m}}\left|Q_{m}(z)\right| \text { for } z \in \partial \mathbb{D}_{R_{m}} .
$$

Then by Rouché's theorem, polynomial $\sum_{k=1}^{m}\left(n_{k} p+1\right)^{1 / p} Q_{k}(z) z^{n_{k}}$ has the same number of zeros as $\left(n_{m} p+1\right)^{1 / p} Q_{m}(z) z^{n_{m}}$ in the disc $\mathbb{D}_{R_{m}}$. Choosing suitably increas- 
ing sequences $\left\{R_{m}\right\}_{m \in \mathbb{N}} \subset(0,1)$ and $\left\{n_{m}\right\}_{m \in \mathbb{N}} \subset \mathbb{N}$ we may guarantee that $f$ has infinitely many zeros in $\mathbb{D}$.

Open Access This article is licensed under a Creative Commons Attribution 4.0 International License, which permits use, sharing, adaptation, distribution and reproduction in any medium or format, as long as you give appropriate credit to the original author(s) and the source, provide a link to the Creative Commons licence, and indicate if changes were made. The images or other third party material in this article are included in the article's Creative Commons licence, unless indicated otherwise in a credit line to the material. If material is not included in the article's Creative Commons licence and your intended use is not permitted by statutory regulation or exceeds the permitted use, you will need to obtain permission directly from the copyright holder. To view a copy of this licence, visit http://creativecommons.org/licenses/by/4.0/.

\section{References}

1. Aleksandrov, A.B.: The existence of inner functions in the ball. Mat. Sb. (N.S.) 118(160), 147-163 (1982)

2. Aleksandrov, A.B.: The existence of inner functions in the ball. Math. USSR Sb. 46(2), 143-159 (1983)

3. Gakhov, F.D.: Boundary Value Problems. Pergamon (1966) (Translated from Russian)

4. Gronwall, T.H.: On the Fourier coefficients of a continuous function. Bull. Am. Math. Soc. 27(7), 320-321 (1920)

5. Karaś, M., Kot, P.: Divergent series of Taylor coefficients on almost all slices. Bull. Belg. Math. Soc. Simon Stevin 26, 1-9 (2019)

6. Kot, P.: On analytic functions with divergent series of taylor coefficients. Complex Anal. Oper, Theory (2017)

7. Kot, P.: Peak set on the unit disc. Technical Transactions Fundamental Sciences 1-NP, pp. 81-84 (2016)

8. Kot, P.: Radon inversion problem for holomorphic functions on strictly pseudoconvex domains. Bull. Belg. Math. Soc. Simon Stevin 17, 623-640 (2010)

9. Løw, E.: A construction of inner functions on the unit ball in $\mathbb{C}^{p}$. Invent. Math. 67, 223-229 (1982)

10. Radon, J.: Über die Bestimmung von Funktionen durch ihre Integralwerte längs gewisser Mannigfaltigkeiten. Berichte über die Verhandlungen der Königlich-Sächsischen Gesellschaft der Wissenschaften zu Leipzig, Mathematisch-Physische Klasse, vol. 69, pp. 262-277 (1917)

11. Rudin W.: Function theory in the unit ball of $\mathbb{C}^{n}$. Reprint of the 1980 edition. In: Classics in Mathematics. Springer, Berlin (2008)

12. Wojtaszczyk, P.: On functions in the ball algebra. Proc. Am. Math. Soc. 85(2), 184-186 (1982)

Publisher's Note Springer Nature remains neutral with regard to jurisdictional claims in published maps and institutional affiliations. 